\title{
A comparison of epidural anaesthesia with lignocaine, bupivacaine and a lignocaine-bupivacaine mixture in cats
}

\author{
$\mathrm{F} \mathrm{M} \mathrm{Lawal}^{\mathrm{a}^{*}}$ and A Adetunji ${ }^{\mathrm{a}}$
}

\begin{abstract}
A mixture of $2 \%$ lignocaine (LIG) and $0.5 \%$ bupivacaine (BUP), at respective dose rates of $2 \mathrm{mg} / \mathrm{kg}$ and $0.5 \mathrm{mg} / \mathrm{kg}$ (LIG-BUP), was compared to LIG (4 mg/kg) and BUP (1 mg/kg) for lumbosacral epidural anaesthesia in 5 sedated cats. Each cat received all 3 treatment regimens at 1 -week intervals. The cats were premedicated with an intramuscular injection of atropine sulphate $(0.04 \mathrm{mg} / \mathrm{kg})$ and ketamine hydrochloride $(10 \mathrm{mg} / \mathrm{kg})$. Onset and duration of analgesia, and time to walking were determined. Associated changes in heart rate $(\mathrm{HR})$, respiratory frequency $\left(f_{R}\right)$ and rectal temperature (RT) were recorded. Onset of analgesia with epidurally administered LIG-BUP (4.5 $\pm 0.7 \mathrm{~min})$, LIG (3.9 $\pm 1.0 \mathrm{~min})$ and BUP $(5.0 \pm 1.0 \mathrm{~min})$ was similar. Duration of analgesia with LIG-BUP $(49.4 \pm 4.5 \mathrm{~min})$ was significantly $(P<0.05)$ longer than with LIG $(40.2 \pm 1.0 \mathrm{~min})$ but shorter than with BUP $(79.4 \pm 6.3 \mathrm{~min})$. There were no significant differences in times to standing with LIG-BUP $(26.3 \pm 6.4 \mathrm{~min}), \mathrm{LIG}(20.4 \pm 4.7 \mathrm{~min})$ and BUP $(22.6 \pm 10.3 \mathrm{~min})$. Minimal changes were observed in HR, $f_{R}$ and RT. In conclusion, duration of analgesia produced by LIG-BUP was shorter compared with BUP but longer compared with LIG. Neither LIG nor LIG-BUP has any advantage over epidural BUP in terms of onset of analgesia, time to standing and physiological responses. Therefore, lumbosacral epidural administration of BUP appears to be the best choice for a long surgical procedure lasting more than 1 hour when compared with either LIG or LIG-BUP.
\end{abstract}

Keywords: Epidural, lignocaine, bupivacaine, ketamine, cats.

Lawal F M, Adetunji A A comparison of epidural anaesthesia with lignocaine, bupivacaine and lignocaine-bupivacaine mixture in cats. Journal of the South African Veterinary Association (2009) 80(4): 243-246 (En.). Department of Veterinary Surgery and Reproduction, Faculty of Veterinary Medicine, University of Ibadan, Ibadan, Nigeria.

\section{INTRODUCTION}

The administration of local anaesthetics into the epidural space is an established technique for producing regional anaesthesia in veterinary practice. Epidural anaesthesia may be used for any surgical procedure caudal to the diaphragm in critically ill animals, particularly where appropriate anaesthetic delivery equipment is not readily available $\mathrm{e}^{10,13,14}$. However, conscious cats often resist handling for clinical procedures without additional chemical restraint and ketamine is frequently used for this purpose ${ }^{10,12}$.

The use of epidural analgesia with lignocaine (LIG), amethocaine, xylazine, medetomidine, morphine and pethidine has been described in the cat ${ }^{1,3,8,18}$. The most frequently used epidural anaesthetic in all species is LIG, although bupivacaine (BUP) is sometimes also used. Whereas LIG is known to have excellent diffusion

aDepartment of Veterinary Surgery and Reproduction, Faculty of Veterinary Medicine, University of Ibadan, Ibadan, Nigeria.

${ }^{*}$ Author for correspondence.

E-mail: fmlawal2002@yahoo.com

Received: August 2009. Accepted: November 2009. and penetrability, as well as produce rapid onset and establishment of surgical anaesthesia, it produces short-duration sensory and motor blockades ${ }^{1,3,10,13,17}$. Used alone, BUP, on the other hand, has slow onset but long duration of action in $\operatorname{dogs}^{2,5,9-11,13,15,17}$. In a busy operating schedule, an agent having the characteristics of fast onset and sufficiently long duration of action would be preferable. This need has led to the use of local anaesthetic mixtures which combine the desirable properties of each component drug. In dogs, LIG and BUP mixture (LIG-BUP) has been shown to produce rapid onset and prolonged duration of analgesia as well as reduced time to standing ${ }^{2,5}$. To date, however, we are not aware of any report of epidural administration of LIG-BUP in the cat.

The aim of this study was therefore to compare LIG-BUP with either LIG or BUP for epidural anaesthesia in terms of time to onset of analgesia, duration of analgesia and time to standing in ketamine-sedated cats not undergoing any clinical procedure.

\section{MATERIALS AND METHODS}

\section{Animals}

Following approval by the Faculty's Institutional Animal Care and Use Committee, 5 healthy, adult Nigerian local cats, comprising 2 intact toms and 3 nonpregnant, non-lactating queens, were studied. The animals were purchased from a local cat market and selection of cats for inclusion in the study was based on American Society of Anesthesiologists physical status 1 (ASA 1). Their body weights ranged from 1.5 to $2.8 \mathrm{~kg}$ (mean \pm SEM $1.9 \pm 0.2 \mathrm{~kg}$ ). The cats were housed individually in catteries and were fed once daily on a home-made, cereal-based concentrate supplemented with fish meal. The cats were allowed to eat ad libitum at each feeding time, while water was freely available. The animals were allowed a period of 4 weeks to familiarise them with their new environment, new feeding regimen and constant human handling. Just before the start of the experiments, the cats were considered to be in good health based on findings at physical examination, haematology and serum chemistry profile.

\section{Study design}

Three drug treatments were carried out at 1 -week intervals on each cat $(n=5)$ in a randomised order. The 1st drug treatment involved lumbosacral epidural administration of LIG, the 2nd treatment involved similar administration of BUP, while the 3rd treatment involved similar administration of LIG-BUP at half the recommended doses of the component drugs. Physiological variables were measured immediately before the epidural injection of the local anaesthetics and subsequently at $10 \mathrm{~min}$ intervals over a period of $100 \mathrm{~min}$. Selected anaesthetic indices were also calculated for each treatment group.

\section{Experimental procedure}

Food was withheld from the cats for 12 hours (i.e. overnight) prior to the experiments, while water was allowed freely until the time of premedication to prevent preanaesthetic dehydration in the cats. 
The body weight of each cat was determined using a special weighing balance with a reading range of $0-20.0 \mathrm{~kg}$. Premedication consisted of the intramuscular injection of $10 \mathrm{mg} / \mathrm{kg}$ of $5 \%$ ketamine hydrochloride (Ketmin, Laborate Pharmaceutical, India) and $0.04 \mathrm{mg} / \mathrm{kg}$ of $0.1 \%$ atropine sulphate (Amopin, Ningbo Chemicals International Trade Corporation, China) mixed together in the same syringe. Following ketamine-induced loss of the righting reflex, the cat was positioned in sternal recumbency. The injection site was located and prepared for aseptic epidural puncture and then the selected local anaesthetic solution was injected into the lumbosacral epidural space as previously described for cats ${ }^{10}$. A dose of either $4 \mathrm{mg} / \mathrm{kg}$ of $2 \%$ LIG (Sensinil, Claris Lifesciences Limited, India), $1 \mathrm{mg} / \mathrm{kg}$ of $0.5 \%$ BUP (Marcain Heavy, Astrazeneca) or $2 \mathrm{mg} / \mathrm{kg}$ of $2 \%$ LIG and $0.5 \mathrm{mg} / \mathrm{kg}$ of $0.5 \%$ BUP mixture (LIG-BUP) was employed at 1-week intervals. The treated cat was maintained in sternal recumbency throughout the period of the experiment. Sensory blockade (analgesia) was assessed by the cat's response to clamping the toe-web with haemostatic forceps closed to the 1st ratchet at 2 min intervals. Whereas the application of a clamp may influence heart rate and respiratory frequency, the clamping was done in each case after the evaluation of these variables.

\section{Measurements}

During each trial, heart rate (HR), respiratory frequency $\left(f_{\mathrm{R}}\right)$ and rectal temperature (RT) were measured immediately before the epidural injection of the drugs (time $0 \mathrm{~min}$ ) and subsequently at $10 \mathrm{~min}$ intervals over a period of $100 \mathrm{~min}$ post injection. Heart rate in beats/min was determined with the aid of a precordial stethoscope. Respiratory frequency was counted in breaths/min by visual observation of thoracic excursions while rectal temperature was measured in degrees Celsius $\left({ }^{\circ} \mathrm{C}\right)$ using a digital clinical thermometer.

\section{Calculations}

For each treated cat, time to onset, duration of analgesia, and time to walking were calculated. These anaesthetic indices were defined as follows: (i) Time to onset of analgesia: time interval (in $\mathrm{min}$ ) between the epidural injection of the drug and loss of response to pain inflicted on the toe-web by the haemostat. (ii) Duration of analgesia: time interval (in min) between loss and reappearance of response to pain inflicted on the toe-web by the haemostat. (iii) Time to standing: time interval (in min) between reappearance of the pedal with-

Table 1: Mean \pm SEM heart rate (beats $/ \mathrm{min}$ ) in $\mathbf{5}$ ketamine-sedated cats given epidural lignocaine (4 mg/kg; LIG), bupivacaine (1 mg/kg; BUP) and a mixture of lignocaine and bupivacaine $(2 \mathrm{mg} / \mathrm{kg}$ and $0.5 \mathrm{mg} / \mathrm{kg}$; LIG-BUP).

\begin{tabular}{rlll}
\hline \multirow{2}{*}{ Time interval (min) } & \multicolumn{1}{c}{ HR (beats/min) } \\
\cline { 2 - 4 } & LIG & BUP & LIG-BUP \\
\hline 0 & $233.6 \pm 15.9^{\dagger}$ & $203.2 \pm 4.3$ & $192.8 \pm 8.5$ \\
10 & $210.4 \pm 11.2$ & $220.8 \pm 13.2$ & $200.8 \pm 8.4$ \\
20 & $220.8 \pm 11.0$ & $216.4 \pm 12.2$ & $207.2 \pm 5.6^{*}$ \\
30 & $210.4 \pm 17.0$ & $226.4 \pm 7.5^{\star \dagger}$ & $206.4 \pm 8.3$ \\
40 & $212.0 \pm 13.3$ & $227.2 \pm 6.4^{\star \dagger}$ & $200.8 \pm 3.9$ \\
50 & $200.0 \pm 8.1^{*}$ & $220.0 \pm 10.4^{\star \dagger}$ & $202.4 \pm 5.6$ \\
60 & $220.4 \pm 10.5$ & $203.2 \pm 14.9$ & $203.2 \pm 9.4$ \\
70 & $226.4 \pm 13.1^{\dagger}$ & $217.6 \pm 7.1^{\star \dagger}$ & $201.6 \pm 7.8$ \\
80 & $212.8 \pm 4.9$ & $214.8 \pm 15.2$ & $202.4 \pm 7.5$ \\
90 & $225.6 \pm 5.2^{\dagger}$ & $208.8 \pm 10.1$ & $193.6 \pm 6.9$ \\
100 & $219.2 \pm 15.3$ & $207.6 \pm 9.2$ & $199.6 \pm 9.5$ \\
\hline
\end{tabular}

*Significantly different from 0 min in each group.

${ }^{\dagger}$ Significantly different from LIG-BUP group $(P<0.05)$

Table 2: Mean \pm SEM respiratory frequency (breaths $/ \mathrm{min}$ ) in 5 ketamine-sedated cats given epidural lignocaine (4 mg/kg; LIG), bupivacaine (1 mg/kg; BUP) and a mixture of lignocaine and bupivacaine $(2 \mathrm{mg} / \mathrm{kg}$ and $0.5 \mathrm{mg} / \mathrm{kg}$; LIG-BUP).

\begin{tabular}{rccc} 
Time interval (min) & \multicolumn{3}{c}{ RR (breaths/min) } \\
\cline { 2 - 4 } & LIG & BUP & LIG-BUP \\
\hline 0 & $31.2 \pm 5.6$ & $34.8 \pm 6.3$ & $26.8 \pm 7.4$ \\
10 & $32.8 \pm 5.6$ & $36.4 \pm 6.6$ & $27.6 \pm 8.2$ \\
20 & $42.0 \pm 6.8$ & $51.2 \pm 10.8$ & $40.4 \pm 9.0$ \\
30 & $34.4 \pm 6.8^{\dagger}$ & $55.8 \pm 12.4^{*}$ & $53.2 \pm 7.6^{*}$ \\
40 & $40.8 \pm 12.1$ & $61.6 \pm 10.1^{*}$ & $53.6 \pm 8.6^{*}$ \\
50 & $47.2 \pm 7.6^{*}$ & $60.0 \pm 10.9^{*}$ & $49.2 \pm 6.0^{*}$ \\
60 & $45.2 \pm 9.9$ & $56.6 \pm 8.8^{*}$ & $56.4 \pm 9.5^{\star}$ \\
80 & $54.0 \pm 8.2^{\star}$ & $48.4 \pm 7.5$ & $61.6 \pm 9.0^{*}$ \\
90 & $48.2 \pm 6.8^{\star}$ & $52.0 \pm 8.1^{*}$ & $52.0 \pm 4.6^{*}$ \\
100 & $44.8 \pm 7.6$ & $60.0 \pm 12.5^{*}$ & $46.0 \pm 5.5^{\star}$ \\
& $40.4 \pm 5.4$ & $61.6 \pm 12.2^{*}$ & $47.8 \pm 6.8^{*}$
\end{tabular}

*Significantly different from 0 min in each group.

†Significantly different from LIG-BUP group $(P<0.05)$

drawal reflex and the cat's ability to stand on its hindlimbs and walk around.

\section{Statistical analysis}

The data are expressed as mean \pm SEM. Anaesthetic indices of the epidurally administered LIG-BUP, LIG and BUP were compared using 1-way ANOVA followed by Bonferroni $t$ procedure when a significant difference was indicated. Physiological variables were compared using analysis of variance (ANOVA) for repeated measures in each group to assess changes from control with time. Dunnett's test was used as post test ${ }^{6}$. For all analyses, a value of $P<0.05$ was considered statistically significant. The statistical package NCSS 2004 (Lange/McGraw-Hill, New York) was used ${ }^{6}$.

\section{RESULTS}

\section{Calculated anaesthetic indices}

The onset of analgesia with epidurally administered LIG-BUP (4.5 $\pm 0.7 \mathrm{~min})$,
LIG (3.9 $\pm 1.0 \mathrm{~min})$ and BUP (5.0 \pm $1.0 \mathrm{~min})$ was similar. Duration of analgesia with LIG-BUP $(49.4 \pm 4.5 \mathrm{~min})$ was significantly $(P<0.05)$ longer than that with LIG (40.2 $\pm 1.0 \mathrm{~min})$ but shorter than that with BUP $(79.4 \pm 6.3 \mathrm{~min})$. There were no significant differences in times to standing with LIG-BUP $(26.3 \pm 6.4 \mathrm{~min})$, LIG $(20.4 \pm 4.7 \mathrm{~min})$ and BUP $(22.6 \pm$ $10.3 \mathrm{~min})$

\section{Measured physiological variables}

The means HR, $f_{\mathrm{R}}$ and RT for each of the 3 treatment groups are shown in Tables 1-3. Mean HR ranged from $192.8 \pm$ 8.5 to $207.2 \pm 5.6$ (beats $/ \mathrm{min}$ ) with epidurally administered LIG-BUP, from $200.0 \pm 8.1$ to $233.6 \pm 15.9$ (beats/min) with LIG and from $203.2 \pm 14.9$ to $227.2 \pm$ 6.4 (beats/min) with BUP. Ranges of the mean $f_{\mathrm{R}}$ for the treatment groups were from $26.8 \pm 7.4$ to $61.6 \pm 9.0$ breaths $/ \mathrm{min}$ (LIG-BUP), from $31.2 \pm 5.6$ to $54.0 \pm 8.2$ breaths/min (LIG) and from $34.8 \pm 6.3$ to $61.6 \pm 12.2$ breaths/min (BUP). Mean RT 
Table 3: Mean \pm SEM rectal temperature $\left({ }^{\circ} \mathrm{C}\right)$ in five ketamine-sedated cats given epidural lignocaine (4 mg/kg; LIG), bupivacaine (1 mg/kg; BUP) and a mixture of lignocaine and bupivacaine (2 $\mathrm{mg} / \mathrm{kg}$ and $0.5 \mathrm{mg} / \mathrm{kg}$; LIG-BUP).

\begin{tabular}{rccc}
\hline \multirow{2}{*}{ Time interval (min) } & \multicolumn{2}{c}{$\mathbf{R T}\left({ }^{\circ} \mathbf{C}\right)$} & LIG-BUP \\
\cline { 2 - 4 } & LIG & BUP & $38.2 \pm 0.5$ \\
0 & $38.7 \pm 0.3$ & $38.3 \pm 0.1$ & $37.9 \pm 0.5$ \\
10 & $38.4 \pm 0.4$ & $38.1 \pm 0.1$ & $37.7 \pm 0.5$ \\
20 & $38.3 \pm 0.4$ & $38.1 \pm 0.1$ & $37.7 \pm 0.5$ \\
30 & $38.1 \pm 0.4$ & $38.0 \pm 0.1^{*}$ & $37.7 \pm 0.5$ \\
40 & $38.1 \pm 0.5$ & $38.1 \pm 0.2$ & $37.7 \pm 0.5$ \\
50 & $38.1 \pm 0.4$ & $38.1 \pm 0.2$ & $37.8 \pm 0.5$ \\
60 & $38.2 \pm 0.4$ & $38.2 \pm 0.2$ & $37.9 \pm 0.5$ \\
70 & $38.5 \pm 0.5$ & $38.2 \pm 0.3$ & $38.0 \pm 0.5$ \\
80 & $38.6 \pm 0.5$ & $38.3 \pm 0.2$ & $38.1 \pm 0.5$ \\
90 & $38.7 \pm 0.4$ & $38.3 \pm 0.2$ & $38.2 \pm 0.4$ \\
100 & $38.9 \pm 0.4$ & $38.4 \pm 0.2$ & \\
\hline
\end{tabular}

*Significantly different from 0 min in each group.

${ }^{\dagger}$ Significantly different from LIG-BUP group $(P<0.05)$

with LIG-BUP ranged between $37.7 \pm 0.6$ and $38.2 \pm 0.6{ }^{\circ} \mathrm{C}$, with LIG between $38.1 \pm 0.4$ and $38.9 \pm 0.4^{\circ} \mathrm{C}$ and with BUP between $38.0 \pm 0.4$ and $38.4 \pm 0.2{ }^{\circ} \mathrm{C}$.

\section{DISCUSSION}

Various factors, such as animal's age, obesity, pregnancy and body posture, are known to influence the spread of anaesthetic solution within the epidural space $^{4,10,13,14}$. For this reason, deliberate efforts were made in this study to exclude paediatric, geriatric, obese and pregnant animals. In clinical practice, epidural block is often performed on heavily sedated or lightly anaesthetised cats because of their tendency to resist handling for even the simplest clinical procedure ${ }^{10,13,14}$. Ketamine $(10 \mathrm{mg} / \mathrm{kg})$ was used in this trial to ensure complete immobility of the cats but without loss of consciousness for humane epidural puncture as reported in a previous, similar study ${ }^{1}$. Ketamine no doubt could have contributed to the analgesic effect obtained. However, since the 3 treatment groups were given equal doses of ketamine, any difference could not be attributable to ketamine. In this study, solutions of LIG and BUP were observed to be miscible in the syringe, indicating pharmaceutical compatibility. It is noteworthy that no evidence of incompatibility of the 2 local anaesthetic solutions was reported in previous similar studies in dogs either ${ }^{2,5}$. This finding suggests that solutions of the 2 local anaesthetics do not differ greatly in their $\mathrm{pH}$ values. Selection of the dose of LIG $(4 \mathrm{mg} / \mathrm{kg})$ and BUP $(1 \mathrm{mg} / \mathrm{kg})$ employed in this study was based primarily on the information provided in the previous studies $^{10,13,17}$. The treated cats were maintained in sternal recumbency throughout the period of the experiment, thereby keeping the spine horizontal for bilateral block, although it is recognised that the sternal position may not be suitable for all surgical procedures. The small number of animals used $(n=5)$ might be responsible in part for the variability in the data obtained, which is likely to disappear with the use of a larger number of animals $(n \geq 30)$.

Times to onset of analgesia with the 3 drug regimens did not differ significantly. This agrees with a similar study of epiBUP in dogs ${ }^{2}$. The authors used $5.7 \mathrm{mg} / \mathrm{kg}$ of $2 \%$ solution (LIG) with 1:100 000 adrenaline and $2 \mathrm{mg} / \mathrm{kg}$ of $0.5 \%$ solution (BUP) in a lumbosacral epidural injection and reported respective onsets of analgesia of $5.0 \pm 0.0 \mathrm{~min}, 6.0 \pm 1.0 \mathrm{~min}$ and $6.4 \pm$ $1.4 \mathrm{~min}$ for LIG, BUP and LIG-BUP. In this study, duration of analgesia of $79.4 \pm$ 6.3 min with a single dose of epidural administration of BUP doubled that with LIG while that with LIG-BUP was intermediate. This is in accord with the data obtained in another similar study in dogs in which $5 \mathrm{mg} / \mathrm{kg}$ of $2 \%$ solution (LIG) with 1:200 000 adrenaline and $1.25 \mathrm{mg} / \mathrm{kg}$ of $0.5 \%$ solution (BUP) with 1:200 000 adrenaline were employed and duration of analgesia of $54.0 \pm 5.0 \mathrm{~min}, 110.0 \pm$ $14.0 \mathrm{~min}$ and $94.0 \pm 8.0 \mathrm{~min}$ were reported for LIG, BUP and LIG-BUP, respectively ${ }^{5}$. This suggests that the component drugs in the LIG-BUP combination had additive effects as has also been reported in humans $s^{7,16}$. The reported onset and duration of analgesia in the studies in dogs appeared to be longer than those recorded in this study. These discrepancies may relate to differences in the animal species studied and higher dosages of drugs employed, as well as the presence of adrenaline in the drug solutions used in the earlier studies. The addition of adrenaline to local anaesthetic solutions is said to prolong their duration of action by decreasing the vascular uptake of the durally administered LIG, BUP and LIG- drug solutions, therebyreducing the possibility of systemic toxicity ${ }^{10,13,17}$. Times to standing were similar with the epidural administration of the 3 drug regimens. This shows that the LIG-BUP has no advantage over the component drugs in terms of recovery period.

In this study, epidurally administered LIG, BUP and LIG-BUP produced minimal changes in the cat's HR, $f_{R}$ and RT both within and between the treatment groups (Tables 1-3). This finding agrees with previous similar studies of epidurally administered LIG in cats and epidural administration of LIG, BUP and LIG-BUP in $\operatorname{dog} \mathrm{s}^{1,2,5}$. Heart rate decreased over time to $50 \mathrm{~min}$ to reach statistical significance with LIG, whereas HR increased to reach statistical significance at $30 \mathrm{~min}$ with BUP. The reason for the difference between LIG and BUP in HR changes in the early part of the trials is not clear. It is likely to be due to the greater ability of LIG to diffuse through the cerebrospinal fluid (CSF) to the brain to cause depression of the cardiovascular centre, making it less responsive to baroreceptor signals of hypotension due to neural blockade. This supposition could not be confirmed in this study as blood pressure was not measured, but it is supported by the results of a previous study ${ }^{5}$.

In conclusion, duration of analgesia produced by LIG-BUP was shorter compared with BUP but longer compared with LIG. Neither LIG nor LIG-BUP has any advantage over epidural BUP in terms of onset of analgesia, time to standing and physiological responses. Therefore, lumbosacral epidural administration of BUP appears to be the best choice for a long surgical procedure lasting more than 1-hour compared with either LIG or LIG-BUP.

\section{REFERENCES}

1. Adetunji A, Adewoye C O, Ajadi R A 2002 Comparison of epidural anaesthesia with lignocaine or xylazine in cats. Veterinary Journal 163: 335-336

2. Adetunji A, Ajadi R A, Aladesawe T A 2001 A comparison of epidural anaesthesia with lignocaine, bupivacaine and lignocaine/ bupivacaine mixture in dogs. Israel Journal of Veterinary Medicine 56: 85-89

3. Adetunji A, Nweke R I, Akinade S A, Ajao O A 2003 Comparison of epidural lignocaine and medetomidine in ketaminesedated cats. Nigerian Veterinary Journal 24: 111-116

4. Bromage P R 1962 Spread of analgesic solutions in the epidural space and their site of action. British Journal of Anaesthesia 34: 361-365

5. Cruz M L, Luna S P L, Clark R M O, Massone F, Castro G B 1997 Epidural anaesthesia using lignocaine, bupivacaine or a mixture of lignocaine and bupivacaine in dogs. Journal of Veterinary Anaesthesia 24: 30-32 
6. Dawson B, Trapp R G 2004 Basic and clinical biostatistics (4th edn). Lange Medical Publication/McGraw Hill, New York

7. DeJang R T, Bonin J D 1981 Mixtures of local anaesthetics are no more toxic than the parent drugs. Anaesthesiology 54: 177-181

6. Duce B R, Zelechowski K, Camougis G, Smith E R 1969 Experimental epidural anaesthesia in the cat with lignocaine and amethocaine. British Journal of Anaesthesia 41: 579-587

9. Feldman H S, Dvoskin S, Arthur G R, Doucette A M 1996 Antinociceptive and motor-blocking efficacy of ropivacaine and bupivacaine after epidural administration in the dog. Regional Anaesthesia 21: 318326

10. Hall L W, Clarke K W, Trim C M 2001 Veteri- nary anaesthesia (10th edn). W B Saunders, London

11. Heath R B, Broadstone R V, Wright M, Grandy J L 1985 Bupivacaine and mepivacaine lumbosacral epidural analgesia in dogs. Proceedings of the 2 nd International Congress of Veterinary Anaesthesia, Sacramento, California, 7-10 October 1985: 162-163

12. Jones R S 1979 Injectable anaesthetic agents in the cat: a review. Journal of Small Animal Practitioners 20: 345-352

13. Jones R S 2001 Epidural analgesia in the dog and cat: a review. Veterinary Journal 161: 123-131

14. Klide A M, Soma L R 1968 Epidural analgesia in the dog and cat. Journal of the American Veterinary Medical Association 153: 165-172

15. Lebeaux M I 1973 Experimental epidural anaesthesia in the dog with lignocaine and bupivacaine. British Journal of Anaesthesia 45: 549-555

16. Seow L T, Lips E J, Cousins M J, Mather L E 1982 Lidocaine and bupivacaine mixtures for epidural blockade. Anaesthesiology 56: 177-183

17. Skarda R T 1996 Local and regional anaesthetic and analgesic techniques: Dogs. In Thurmon J C, Tranquilli W J, Benson G J (eds) Lumb and Jones' veterinary anaesthesia (3rd edn). Williams and Wilkins, Baltimore: 434-447

18. Tung A S, Yaksh T L 1982 The antinociceptive effects of epidural opiates in the cat: studies on the pharmacology and the effects of lipophilicity in spinal analgesia. Pain 12: $343-356$ 